\title{
Designing a Secure Blue Button Health Information Exchange for a Commercial Laboratory
}

Seth Baker, CPHIMS, PMP, MCSE, MCSD; Alex Roman, BS, MS

Hartford Healthcare / Clinical Lab Partners, Newington, CT, United States

Corresponding Author:

Seth Baker, CPHIMS, PMP, MCSE, MCSD

Hartford Healthcare / Clinical Lab Partners

129 Patricia M. Genova Dr.

Newington, CT, 06111

United States

Phone: 18606968614

Fax: 18606968620

Email: sbaker@arbot.com

\section{Abstract}

Background: There is a growing demand by patients for easy electronic access to laboratory result data for use in personal health record systems (PHR-S) and for transfer to other health providers. The Blue Button Initiative is a public-private partnership offering a framework based on national standards to support patient access to electronic data.

Objective: The aim of this project study was to architect an initial pilot implementation of the Blue Button framework for a commercial laboratory to facilitate patient access to electronic results.

Methods: The proposed design architecture includes multiple application services, specifically an Encrypted Data Store, Client Access component, and Result Publishing service to accomplish these goals of the pilot project and meet the security and privacy requirements.

Results: The resulting application components and programming interfaces accomplish the initial pilot goals and provide a base to expand the platform to offer support for mobile devices and additional interoperability options. Encryption and isolation of data have been used to safeguard the confidentiality, integrity and availability of protected health information (PHI) and allow for the use of standard cloud services to host external facing components.

Conclusions: The Blue Button standards and framework provide a solid basis for facilitating electronic access to result data by patients and for meeting the requirements of View, Download, and Transmit (V/D/T) in Meaningful Use Stage 2 (MU-II). The Blue Button Framework can provide the functionality required for a Consumer Mediated Health Information Exchange which gives patients the ability to aggregate and control the use of their health information among providers.

(iProc 2015;1(1):e7) doi: 10.2196/iproc.4685

\section{KEYWORDS}

Blue Button; Blue Button +; Blue Button Plus, health information exchange

(This is a conference paper presented at the Connected Health

Symposium, Boston, 2015, which was not edited and is only lightly peer-reviewed).

\section{Multimedia Appendix 1}

Extended abstract.

[PDF File (Adobe PDF File), 738KB-Multimedia Appendix 1] 
Edited by G Eysenbach, T Hale; submitted 14.05.15; peer-reviewed by D Klein, L Garvin, K Nazi; accepted 20.07.15; published 27.10.15

Please cite as:

Baker S, Roman A

Designing a Secure Blue Button Health Information Exchange for a Commercial Laboratory

iProc 2015;1(1):e7

URL: http://www.iproc.org/2015/1/e7/

doi: 10.2196/iproc.4685

PMID:

(CSeth Baker, Alex Roman. Originally published in JMIR Mhealth and Uhealth (http://www.iproc.org), 27.10.2015. This is an open-access article distributed under the terms of the Creative Commons Attribution License (http://creativecommons.org/licenses/by/2.0/), which permits unrestricted use, distribution, and reproduction in any medium, provided the original work, first published in JMIR mhealth and uhealth, is properly cited. The complete bibliographic information, a link to the original publication on http://mhealth.jmir.org/, as well as this copyright and license information must be included. 